Classification

Physics Abstracts

$6116 \mathrm{D}-0780$

\title{
Approaches for energy loss and energy filtered imaging in TEM in relation to the materials problems to be solved
}

\author{
William Michael Stobbs and Christopher Brian Boothroyd \\ Department of Materials Science and Metallurgy, Cambridge University, Pembroke St., Cambridge \\ CB2 3QZ, G.B.
}

(Received August 20, 1990; accepted March 26, 1991)

\begin{abstract}
The requirements of an energy filtering system for a transmission electron microscope (TEM) are enumerated in relation to the nature of the problems that could be tackled in materials science, if an instrument of sufficient quality were available. Accordingly, we describe the principles for a design for such a system, as based on a $\pi / 2$ spectrometer with additional imaging quadrupoles and lenses, in relation to the different classes of problems for which it would be used. We further describe in more detail the changes in the design of a magnetic sector which are required when it is to be used primarily for imaging rather than for spectroscopy.
\end{abstract}

\section{Introduction.}

One of the most simplistic, and apparently reasonable, assumptions which is normally made in relation to instrumentation for electron microscopy is that the ultimate performance in any specific application, as in for example the areas of high resolution structure characterisation or of localised compositional analysis, must require a specialised design: the general purpose instrument is thus expected in some way to be a compromise. In this context it is normally considered that a probe approach with a scanned beam, as in a specialised STEM, is needed for the best analysis (whether by energy dispersive $\mathrm{x}$-ray spectroscopy (EDXS) or by parallel electron energy loss spectroscopy (PEELS) methods) and that the best high resolution TEM configuration, whether using a symmetric or asymmetric objective in conjunction with a cylindrically symmetric specimen holder configuration, will preclude useful EDXS methods and makes PEELS probe techniques difficult without a compromise in the lens characteristics. We will argue here that the specialist instrument in either form is less useful than might have been expected, and describe how it is that if we first consider the need for the quantitative interpretation of the data for the solution of any given materials problem, it transpires that it is a specific form of general purpose TEM which is necessary if usefully quantitative results are to be obtained.

The design requirements of the TEM based instrument which we have come to favour include a capability to form both energy filtered and energy loss images in TEM coupled with the further capability of obtaining PEELS data for reasonably small (but not atomically dimensioned) specimen areas. From a point of view originating in the needs of practical problems in materials 
science, and bearing in mind the beam spread in a thin foil (even for PEELS), probe sizes in a TEM based $\left(\mathrm{LaB}_{6}\right.$ gun) instrument need not be less than about $3 \mathrm{~nm}$ (particularly if, as discussed below, "analysis" can be contemplated in the TEM image as either filtered or formed using specific loss electrons). In order to understand why it is that we feel that, perforce, this is the type of instrument most suited for materials science we will need to follow through the major problems which are currently beginning to be appreciated in the quantitative analysis of high resolution TEM images as used for the characterisation of structural inhomogeneities and these are thus summarised in section 2 . In section 3 we discuss why it is that an "add on" $\pi / 2$ spectrometer based system is probably the most appropriate for an imaging filter and give our own design approach further detailing changes in the design of the magnetic sector which appear to be desirable given its usage as part of an image chain. We demonstrate finally why the need for such instrumentation is so acute summarising in section 4 the classes of materials problem which could then be attempted.

\section{Why a filter is necessary for quantitative TEM.}

Transmission Electron Microscopes have been widely, and increasingly, used in materials science for the characterisation of localised inhomogeneities for more than forty years and one of the major strengths of the approach seems to be the way in which new techniques for their use come to be developed as new problem areas are appreciated. The longevity of the basic method is however in some ways a danger in that it can become difficult to escape from an accepted approach. We believe that a case in point is the way in which it is generally considered that high resolution imaging must provide the best data on a localised structural inhomogeneity, while it is the inelastic scattering which has to be analysed (whether by the use of EDXS or EELS methods) using a localised probe if an area is to be chemically analysed. The spatial resolution of a probe method for compositional analysis is always in principle limited by beam spread, but the more important and fundamental point worth remembering is that the elastic cross-sections are as characteristic of the elements present as are the inelastic cross-sections. Accordingly the intensity distribution in an elastic image contains information that must be usable in an analysis and to a resolution comparable to that in a through focal image series. The non-linearities associated with dynamic scattering can be modelled easily and fully quantitatively given that there are no problems with the effects which are associated with the elastic scattering of electrons which have undergone energy losses. Ourmazd et al. [1] have appreciated the principle that the elastic image contains chemical information in their approach to the use of quantifications of the patterns in high resolution images for the characterisation of the compositional abruptness of an interface. Even if there are difficulties in the details of some of the arguments used (particularly in relation to the Fresnel effects at interfaces) (e.g. [2]), the fundamental idea is of course sound. We have argued for some time that the Fresnel contrast at a boundary (causing problems in the Ourmazd approach) can itself be quantified to allow the compositional analysis of the boundary to a spatial resolution exceeding that in any of the individual TEM images used. Consequently we have applied the Fresnel Method [3] to problems ranging from grain boundary analysis in ceramics [4] to grain boundary segregation at the atomic level [5] while showing that even the profile of the constituents at both oxide [6] and semiconductor interfaces [7] can be analysed by the approach. In our most recent review of the Fresnel Method [8] as applied to the analysis of the intermediate oxide at the silicon/silica interface [9] we have discussed how its use for compositional analysis is limited by uncertainties in the contribution from inelastically scattered electrons. Fortunately, the analysis of the profile of a composition change at an interface is less affected than is the analysis itself and (for a uniform layer in projection) can be accomplished to atomic, or near atomic, resolution perpen- 
dicular to the boundary of interest. It remains clear however that, if the TEM images used could be filtered, it would become relatively easy to improve the accuracy of a compositional analysis without recourse to the rather difficult techniques which can otherwise sometimes be required in the refinement of the Fresnel based approach when it is used quantitatively for analysis [8]. We do not of course contend that the Fresnel Method should replace STEM based probe methods for analysis and these are still needed to determine the nature of the elements giving rise to the Fresnel effects: the Fresnel Method is complementary to these methods in that it readily allows a spatial resolution rather better than is normally achievable using a probe while equally being of only rather limited value for coarse scale heterogeneities easily characterised using probes. On the other hand probe approaches using a high angle dark filed detector do, excitingly, offer atomic level resolution with sensitivity of the contrast to the atomic number (e.g. [10]) but, so far as we are aware, there remain problems here too with the matching of the intensities observed to those predicted though in this case it is not apparent that filtering is required.

It is significant that in the approach noted above to analysis, suggested by Ourmazd et al. [1], it is the patterns rather than the absolute intensities in the high resolution TEM images that are used. Indeed it is symptomatic of an apparently generally defeatist attitude to the use of high resolution imaging for structural characterisations that, in the analysis of images by their comparison with simulations computed for model structures, the absolute intensities are practically never given and, to our knowledge, have never been matched to any reasonable accuracy. The problem in relation to high resolution imaging is that electrons which are inelastically scattered do not just contribute to a uniform background but can contribute image detail at comparable or finer resolution than is exhibited in the purely elastic image. The problem is exacerbated for higher resolutions in that high accelerating voltages then tend to be used and, for a given energy loss, the associated inelastic image will be at a smaller overfocus the higher the voltage. Basic aspects of the problem have been described by Stobbs and Saxton [11] and we have demonstrated $[12,13]$ experimentally that, for centre-stop imaging, even qualitative aspects of the high resolution images obtained can not be interpreted without the incorporation in the image simulation of contributions from the inelastically scattered electrons. It should perhaps be emphasised that the inclusion of "absorption" in a multislice simulation does not deal with the problem and if indeed the inelastic scattering is treated properly in the theoretical simulation then the "progressive loss of image intensity with specimen thickness and as a function of orientation" (absorption) which occurs would have to be treated in a different way than it normally is. A theoretical treatment of the inelastic contribution for a typical application of high resolution TEM, the analysis of the level of oxygen ordering in $\mathrm{YBa}_{2} \mathrm{Cu}_{3} \mathrm{O}_{7-\delta}$, has shown that the magnitude of the problem is such as to negate the use of high resolution imaging in such a characterisation for, for example, the analysis of the degree of oxygen ordering if the inelastic contributions can not either be filtered out or included uniquely in the analysis [14]. The difficulty here, as in all other image analysis problems for crystalline material, is that it is far from clear whether the inelastic contribution should be treated in the coherent or incoherent limit. While for low angle, intraband, scattering Howie demonstrated several years ago that it is the coherent limit which should be used [15], it remains clear that there will be at least some contributions to the atomic detail in the image in the incoherent limit as associated with interband, and higher angle, scattering; what the relative fractions of these two contributions should be in each of the rather different resultant inelastic images to be incoherently added to the remnant elastic image remains problematic! It is this which makes the inclusion of these effects into high resolution image simulations rather difficult to do without a wealth of further experimental detail on the relative amounts of each type of contribution, particularly when it is remembered that the ratio of the coherent to the incoherent behaviour will vary across a defect.

In our view then the quantitative use of high resolution images for the structural characterisa- 
tion of local inhomogeneities, particularly if localised displacements are required to fractions of the close packed planar spacings, requires either the understanding and inclusion of the inelastic contribution or its removal. If we further note the non-linearity of the fitting procedure it is clear that we are forced into a necessity to filter the image if we are not to have uncertainties in the uniqueness of our analysis. If we have gone to this extreme and incorporated an image filter in our microscope allowing atomic resolution (as has already been demonstrated to be practicable (e.g. [16])) we should clearly now consider whether or not it might be preferable to obtain useful compositional information at a very high resolution in a loss image in TEM rather than by using a probe in STEM. The problem has been discussed in detail elsewhere [17] and it is interesting that at any resolution it seems that there are theoretical advantages in using TEM rather than a STEM for the analysis of an area 100 by 100 times the spatial resolution required. Whether or not these theoretical advantages could be realised in practice, bearing in mind the rather protracted imaging times that would be needed using either method (particularly for higher energy losses with low cross-sections), remains to be seen. It must however remain true that on a TEM approach the well known damaging problems associated with using a small probe at a high current-density for a STEM based image would be obviated. If the current density in the probe were to be reduced from its optimum value to prevent such an effect when using STEM then the relative time advantage of the TEM loss image over that constructed using the probe would be still greater. On the basis of the above discussion it is noteworthy that we seem still to be intent on using inelastic scattering effects for analysis. Generally this will almost certainly be a less efficient method of obtaining such an analysis than will be the quantification of the intensities as a function of thickness in the filtered elastic image given that the nature of the elements present is known from qualitative energy loss imaging (hopefully still in TEM!).

In summary we have argued here that a filter is a necessity for full quantification of a high resolution image for a structural analysis. If such a facility is available it throws into question the use of a conventional probe for localised compositional analysis. We would not argue that probe methods would cease to be needed (particularly for CBED etc) but it is clear that, at the minimum, alternative methods would compliment the probe approach given the use of a filter. The approach we discuss here is far from new: imaging filters have been available at Toulouse for perhaps thirty years and the Zeiss 902 is a commercially available instrument which can be used in the ways described, even if in a format suitable in the main for biological rather than materials applications. Even so, Reimer et al. [18-20] have recently gone a long way, using a Zeiss 902, to demonstrate the potential of quantitative spectroscopic imaging in TEM for a variety of potential applications. There was also a considerable amount of work in the mid 60's on the use of energy analysed line images obtained using Möllenstedt analysers (e.g. [21,22]) which it could be argued presaged most of the ideas above. It is thus more surprising that the approaches to quantitative imaging using filtering that we discuss here in the high resolution context are taking so long to be generally used even if they are increasingly becoming appreciated and quantified $[11,18-20]$.

\section{An appropriate energy filter design.}

Möllenstedt filters, like Castaing-Henry mirrors, require a second high voltage stabilised input in to the column and thus would, with current technology, add more problems in high resolution imaging than would be solved. The symmetry of the $\Omega$ filter is highly attractive and Rose et al. [23] have demonstrated that its aberrations can be limited to a level which would appear to be better than has as yet been achieved by any other method. Unfortunately such a filter is conventionally incorporated in the column immediately after the objective lens and, together with the extra lens needed for the low aberration configuration, leads to a column height which would tend to be 
inconvenient. The $\Omega$ configuration can also hinder alternative usage of the microscope and would, perhaps more importantly, be rather difficult to add to an existing machine. On the other hand there has been a considerable amount of effort put into in the design of $\pi / 2$ spectrometers (e.g. [24-27]) and, even though the principles of the design optimisation for a spectrometer are rather different than for a filter, the experience gained in the former area is proving useful in the latter. We gather that Gatan have now, for example, extended the design of their $\pi / 2$ spectrometer with the incorporation of sextupoles as well as quadrupoles [28] both to eliminate aberrations not accessible in the Rose treatment for an $\Omega$ filter and to improve its imaging capability.

That Gatan are now to market an imaging spectrometer of an "add-on" format, has led to us stopping the development work we have been doing on the development of our own earlier PEELS system [27] for imaging. However the approach we have been taking to the design of such a filter has been based rather specifically on its potential needs and for this reason we give below some of our conclusions on an appropriate configuration. It would be perfectly practicable to do both the image formation and probed energy spectrum acquisition at the end of a $\pi / 2$ spectrometer and quadrupole chain and this is, we gather, the approach which is currently being taken by Gatan [28]. However we have felt it to be important to retain a simpler understanding of what different elements of this chain are doing than such an approach would easily allow. This is because the major problem in the design of a filter based on a $\pi / 2$ spectrometer with extra quadrupoles is that the more the dispersion of a given element, the greater its rectangular distortion. Unfortunately the distortion of the image is not just an inconvenience. The loss of symmetry, and the variations of the phase changes for different orientations in the image plane would distort the intensity distributions in different directions in the image of even a perfect crystal. For an unknown structural defect it would no longer be possible to model the image characteristics in a unique manner. This would clearly defeat the object of having the filter in the first place in that the primary application for which the filter is required, at least in the high resolution field, is to solve the uniqueness problem in relation to the modelling of an image, as is associated with the incorporation of detail in an unfiltered image due to the elastic scattering of energy loss electrons. A primary design principle of the configuration we have examined has thus been to separate the part of the filter producing the dispersion (and phase distortions) from a second quadrupole group the purpose of which is to remove the rectangular distortion caused by the magnetic sector as well as by the quadrupole grouping prior to the energy selection point. Once rectangular distortion is eliminated the maintenance of a short imaging path for an "add-on" spectrometer to a column requires the use of a pancake projector lens for the final image magnification (given that a cylindrically symmetric system is then to be preferred). Image rotation caused by this lens is not a problem and could in any case be cancelled by suitable matching with the microscope column intermediate lens conditions.

When we made a PEELS system some time ago [27] we noted the importance of being able to retain the ability to do conventional serial EELS (as well PEELS) in that many materials problems require data obtained by the former method, if not by both. We are clear here that even if the spectrometer as a whole is optimised for the formation of a filtered image it will also be essential to be able to use it for the formation of a spectrum from a probed area. It would also be desirable to be able to examine a spectrum for the area imaged either as filtered, or using an edge, with minimal disruption of the filter image chain and the ability to do this follows naturally from the separation of dispersive and distortion correction parts of the system. Under some circumstances, as perhaps for the evaluation of segregation at a grain boundary, it would also be useful to be able to form an energy analysed line image (as is conventionally obtained using a Möllenstedt system $[21,22])$.

The design of the system shown schematically in figure 1 is accordingly based on the premises firstly that vertical and horizontal phase asymmetries caused by the filtering process should both be understood and, as far as possible, controllably cancelled and secondly that energy loss spectra 


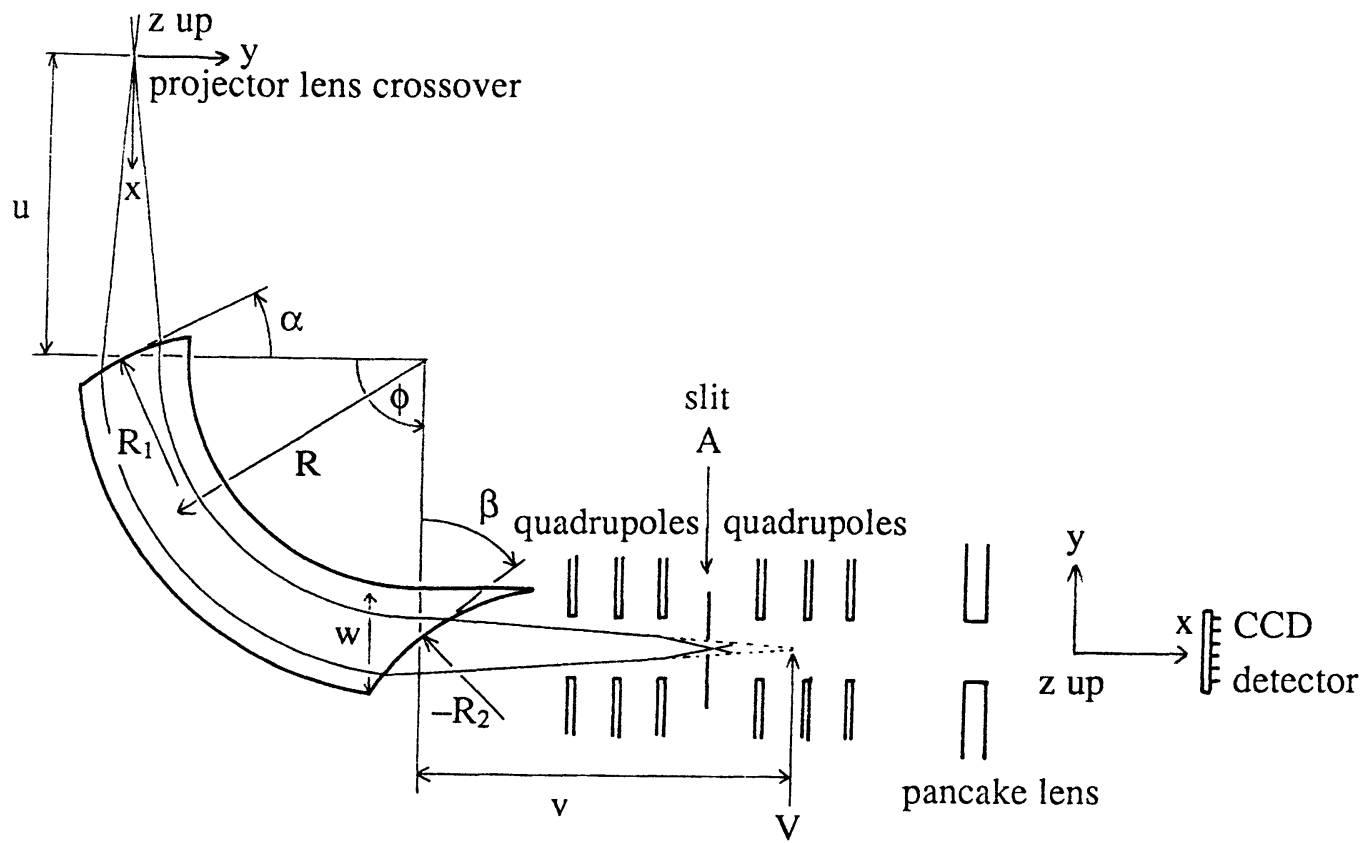

Fig. 1. - Diagram of the filtering system. $u$-object distance $(900 \mathrm{~mm}$ for all the simulations), $v$-image distance of sector alone (ie with quadrupoles off), $\alpha$-entrance tilt angle, $\beta$-exit tilt angle, $R_{1}$ entrance radius, $R_{2}$-exit radius (shown negative here as used for a conventional EELS spectrometer with low $\alpha$ and high $\beta$ ), $R$-sector radius, $\phi$-sector angle ( $90^{\circ}$ for all the simulations), V-position of image of projector crossover with quadrupoles off, A-position of energy selecting slit at image of projector crossover with quadrupoles on.

should also be obtainable (even if not at the energy loss resolutions which can be attained for a system optimised for this purpose), without altering the imaging chain, by displacing the beam to the side of the energy selection slit on to a CCD. The fundamental design of the system shown thus retains the idea that with the diffraction pattern to be filtered in the entrance pupil of the magnetic sector at the microscope projector lens cross over, and with energy loss selection in the necessarily distorted diffraction pattern as tranferred to A (Fig. 1), the asymmetric phase changes caused by the $\pi / 2$ spectrometer and the three quadrupoles before the optic axis slit should be controllably cancelled by the quadrupole system beyond this. Three rather than just two quadrupoles are needed before the slit since we need not only to shorten the imaging arm of the $\pi / 2$ magnetic sector (its image at the distance $v$, providing the virtual object for the first three quadrupoles) but also to allow a variable dispersion when obtaining focussed energy loss spectra on a CCD which would be placed to one side of the optic axis at A. Both for image filtering, as well as for the formation of a loss image using an edge, the slit width need not be better than would allow about a 5 Volt energy window. It is, for example, doubtful whether effects due to smaller voltage differences would be detectable in a filtered high resolution image [11] and for most loss images one would tend to use a still broader window to optimise the number of electrons used to form the image. In this context we, of course, take it that the final loss image would require the subtraction of a background determined from the images obtained using two adjacent windows prior to the edge. Under such circumstances the best window width would normally be about 30 Volts or so but could be as large as $100 \mathrm{~V}$. Accordingly it is interesting that it is important (in a way that is not otherwise obvious) to minimise the chromatic aberration effects of the imaging both beyond and 
before the slit. For an optimised quadrupole system we have found that its imaging characteristics need not be limited by an energy spread of 20 Volts, and should still be adequate for the lower resolutions generally required for a loss image using electrons with a greater energy spread.

On the above principles it is clear that it is appropriate to optimise each of the elemental groups separately and while the system as a whole is described only in principle we consider below the problems associated with the magnetic sector when this is to be used primarily for imaging in rather more detail because the approach required is different from that normally applied when optimising a sector magnet as a spectrometer. It must now form a point focus rather than the line focus used for EELS. This requires double focussing, ie focussing in both the radial $(y)$ and azimuthal ( $z$ ) directions (see Fig. 1), while features such as high dispersion and retention of focus at high dispersion, important for energy loss spectrometers, are less important here. It was required that the system could retain a resolution in the final image of about $0.2 \mathrm{~nm}$ with a $5 \mathrm{eV}$ slit width as would be used for energy filtering of high resolution images and about $0.5 \mathrm{~nm}$ with a $100 \mathrm{eV}$ slit widths as used to obtain images of core loss edges. The system we examined was designed relative to the Cambridge HREM for which the normal operating voltage is $500 \mathrm{kV}$ and, with the spectrometer mounted beneath the microscope screen, the projector-crossover (where the object for the sector is the demagnified diffraction pattern) to spectrometer entrance distance ( $u$ in Fig. 1) is about $900 \mathrm{~mm}$. Ideally it would be possible to pass the image on the screen, at the magnification $(\sim 600 \mathrm{k})$ normally used to set up and align it, through to the spectrometer by simply raising the screen with energy selection at the diffraction pattern at $\mathrm{A}$ as transferred from the point $\mathrm{V}$ to the slit by the first three quadrupoles. However at $600 \mathrm{k}$ an image containing, for example, $1000.2 \mathrm{~nm}$ lattice fringes would be $12 \mathrm{~mm}$ across, which is too large for any spectrometer to cope with. Energy loss spectrometers usually have entrance apertures of 3 to $5 \mathrm{~mm}$ and thus the magnification must be reduced to no more than about $100 \mathrm{k}$ while, depending on the magnification to be applied in the filter, the optimum microscope magnification could be expected to be still lower. Considering the situation at $100 \mathrm{k}$ however the diffraction maxima at the projector crossover, for a resolution of $0.2 \mathrm{~nm}$, are at about $7 \times 10^{-8}$ rad relative to the screen and the object diffraction pattern for the magnetic sector is negligibly small $(\sim 120 \mathrm{~nm})$ and can be taken to be a point source.

In order to obtain double focussing from a magnetic sector the parameters that can be varied are the entrance $(\alpha)$ and exit $(\beta)$ tilt angles, the entrance $\left(R_{1}\right)$ and exit $\left(R_{2}\right)$ radii and the image distance $(v)$. The object distance $(u)$ is fixed to $\sim 900 \mathrm{~mm}$ by the crossover to sector distance and the sector radius $(R)$ was taken to be fixed in our case to $\sim 300 \mathrm{~mm}$ by the space available. Following Shuman $[24]\left({ }^{1}\right)$, first order focussing (considering only first order terms in (1)) gives a quadratic indicating two sets of values for the tilt angles ( $\alpha$ and $\beta$ ) for a given image distance $(v)$. These tilt angles are plotted in figure 2a for the "normal" solution (with a low entrance angle as used in most energy loss spectrometers) and in figure $2 \mathrm{~b}$ for the "alternative" solution (high entrance angle). The exit angle $(\beta)$ is limited to being less than about $45^{\circ}$ since otherwise, with any reasonable exit curvature $\left(R_{2}\right)$, the usable width of the sector is limited too much. Thus reasonable image distances lie between $\sim 350 \mathrm{~mm}$ and $\sim 420 \mathrm{~mm}$ for both the "normal" and "alternative" solutions.

Following Shuman [24] again to consider the second order terms in the imaging behaviour of the sector magnet:

$$
\begin{aligned}
& y_{i}=Y_{1} y_{0}^{\prime}+Y_{2} \delta+Y_{3} y_{0}^{\prime} \delta+Y_{4} y_{0}^{\prime 2}+Y_{5} \delta^{2}+Y_{6} z_{0}^{\prime 2} \\
& z_{i}=Z_{1} z_{0}^{\prime}+Z_{2} y_{0}^{\prime} z_{0}^{\prime}+Z_{3} z_{0}^{\prime} \delta
\end{aligned}
$$

$\left({ }^{1}\right)$ For unwary amateurs such as ourselves it might be noted that in [24] equation A5 for the quadratic in $\tan \alpha$ for first order focusing is wrongly transcribed (but can easily be corrected), as is $y^{\prime} / z^{\prime} z$ in table I, from Enge [29] while Egerton's [30] expression (2.6) for the dispersion $D$ should have $\varepsilon_{2}$ and $\varepsilon_{1}$ transposed in the first but not the second term in the denominator. 


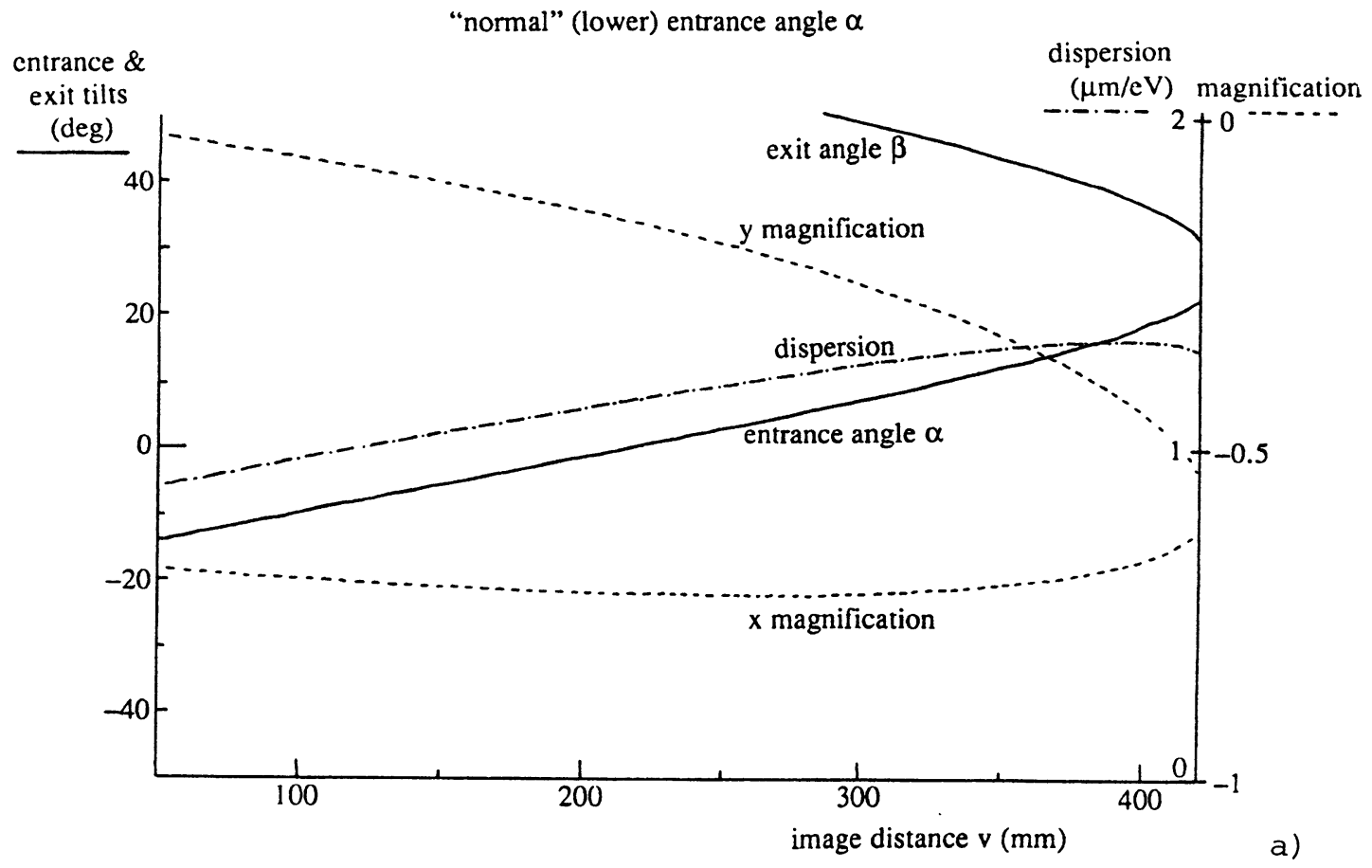

"alternative" (higher) entrance angie $\alpha$

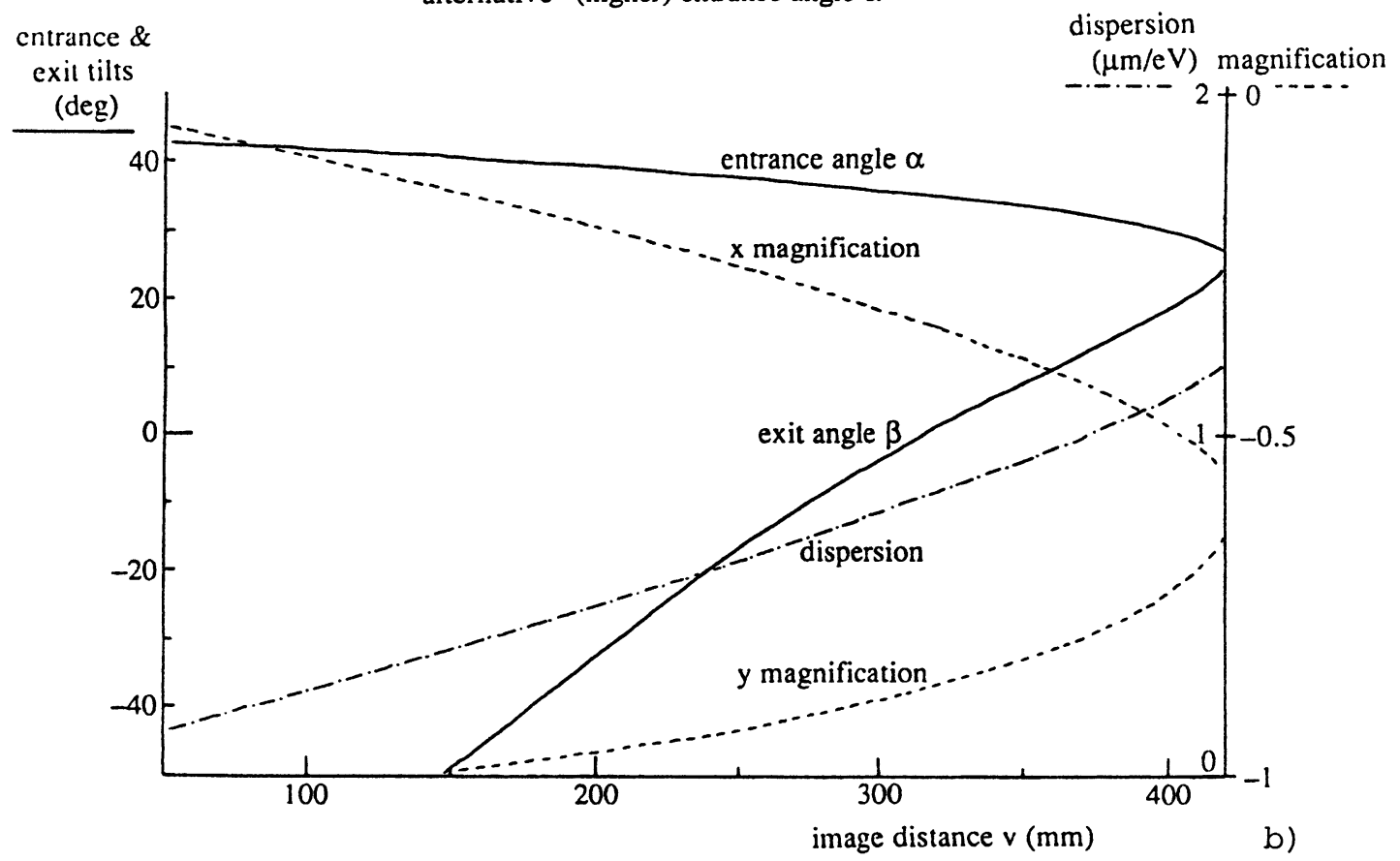

Fig. 2. - Entrance $(\alpha)$ and exit $(\beta)$ tilt angles, dispersion and magnifications plotted as a function of image distance $(v)$ for a sector alone with a) the "normal" (lower) entrance angle $(\alpha)$ and b) the "alternative" (higher) entrance angle $(\alpha)$. 
where the first term of each equation is the first order term considered above. For imaging we are less worried about the terms involving dispersion $\left(Y_{3}, Y_{5}\right.$ and $\left.Z_{3}\right)$ but are as concerned about the other $y$ and $z$ focus terms $\left(Y_{4}, Y_{6}\right.$ and $\left.Z_{2}\right)$, given now that the $z$ focussing terms are important. Because there are only two parameters which can be varied to alter these values, the entrance and exit radii $\left(R_{1}\right.$ and $\left.R_{2}\right)$, only two terms can be made zero, here we have chosen $Y_{4}$ and $Z_{2}$. The resulting radii are plotted in figure 3 . The exit radius, $R_{2}$, is negative, indicating a concave surface, so there is thus a limit on the smallest possible value of $R_{2}$ for a given exit tilt $(\beta)$. For a drift tube half width of $20 \mathrm{~mm}$ and an exit tilt of $\sim 40^{\circ} R_{2}$ has to be greater than about $60 \mathrm{~mm}$. It can be seen from figures 2 and 3 and table I that reasonable values of $R_{1}$ and $R_{2}$ are obtained for the "normal" (low $\alpha$ ) solution, when the image distance is between 380 and $420 \mathrm{~mm}$. This is however where we can also see why the "alternative" lower dispersion (high $\alpha$ ) solution has potential advantages for imaging in that, for above about $350 \mathrm{~mm}, R_{2}$ is positive and large.

Table I. - Entrance and exit pole face angles and curvatures for three image distances for the "normal" solution (low $\alpha$ ). In all three cases the dispersion is about $1.3 \mu \mathrm{m} / \mathrm{eV}$.

\begin{tabular}{|c|c|c|c|c|c|}
\hline $\begin{array}{c}\text { image } \\
\text { distance } \\
v(\mathrm{~mm})\end{array}$ & $\begin{array}{c}\text { entrance } \\
\text { angle } \\
(\alpha)\end{array}$ & $\begin{array}{c}\text { exit } \\
\text { angle } \\
(\beta)\end{array}$ & $\begin{array}{c}\text { entrance } \\
\text { curvature } \\
R_{1}(\mathrm{~mm})\end{array}$ & $\begin{array}{c}\text { exit } \\
\text { curvature } \\
-R_{2}(\mathrm{~mm})\end{array}$ & $\begin{array}{c}\text { drift tube } \\
\text { half width } \\
w / 2(\mathrm{~mm})\end{array}$ \\
\hline 380 & 16 & 40 & 347 & 69 & 25 \\
\hline 400 & 18 & 37 & 325 & 105 & 41 \\
\hline 420 & 23 & 32 & 260 & 138 & 65 \\
\hline
\end{tabular}

The preceding calculations are useful in giving an idea of the optimum parameters for the sector and how they relate to each other. In order to optimise the sector and to determine the influence of parameters not considered above, ray tracing calculations were performed, for which we are grateful to $\mathrm{M}$ Kato and $\mathrm{K}$. Tsuno for their help with the programs used. The programs trace the path of electrons from an object at the microscope's projector lens crossover, through the sector, considering aberrations up to third order in the radial $(y)$ plane and second order in the azimuthal $(z)$ plane. It is also possible to include quadrupole lenses in the system, but this is not done here. The object at the microscope projector lens cross-over is simulated by nine cones of electron trajectories, eight of which are situated around a square at the very large spacing (by comparison with the size of the diffraction pattern there for the probable microscope magnifications to be used) of $10 \mu \mathrm{m}$ while the ninth is at the centre, as shown in figure 4. Examples of the output from the program, which shows the forms that the spread in the "image point" of the $\pi / 2$ sector magnet take for each of the cones, after the aberration effects of the spectrometer, are shown in figure 5 , in this case for monochromatic electrons. The $\alpha \beta$ configuration which is normally used for a spectrometer designed for energy loss analysis has a low entrance angle $\alpha$ and a high exit angle $\beta$. The lower dispersion solution (high $\alpha$ ) would seem from our analysis (compare Figs. 5 and 6) to have potentially better imaging characteristics given that a high dispersion is not required. Thus for a fixed object distance and bending radius, entrance and exit tilts and radii can be found to provide acceptable point focussing for a range of image distances $(v)$ from $\sim 380$ to $\sim 430 \mathrm{~mm}$. We have also investigated the extent to which there are problems in the effects of a given energy spread for both configurations. The effects of a -10 Volts spread in the input energy are shown for each of the two types of spectrometer in figures 7 and 8 where here we are interested in the 


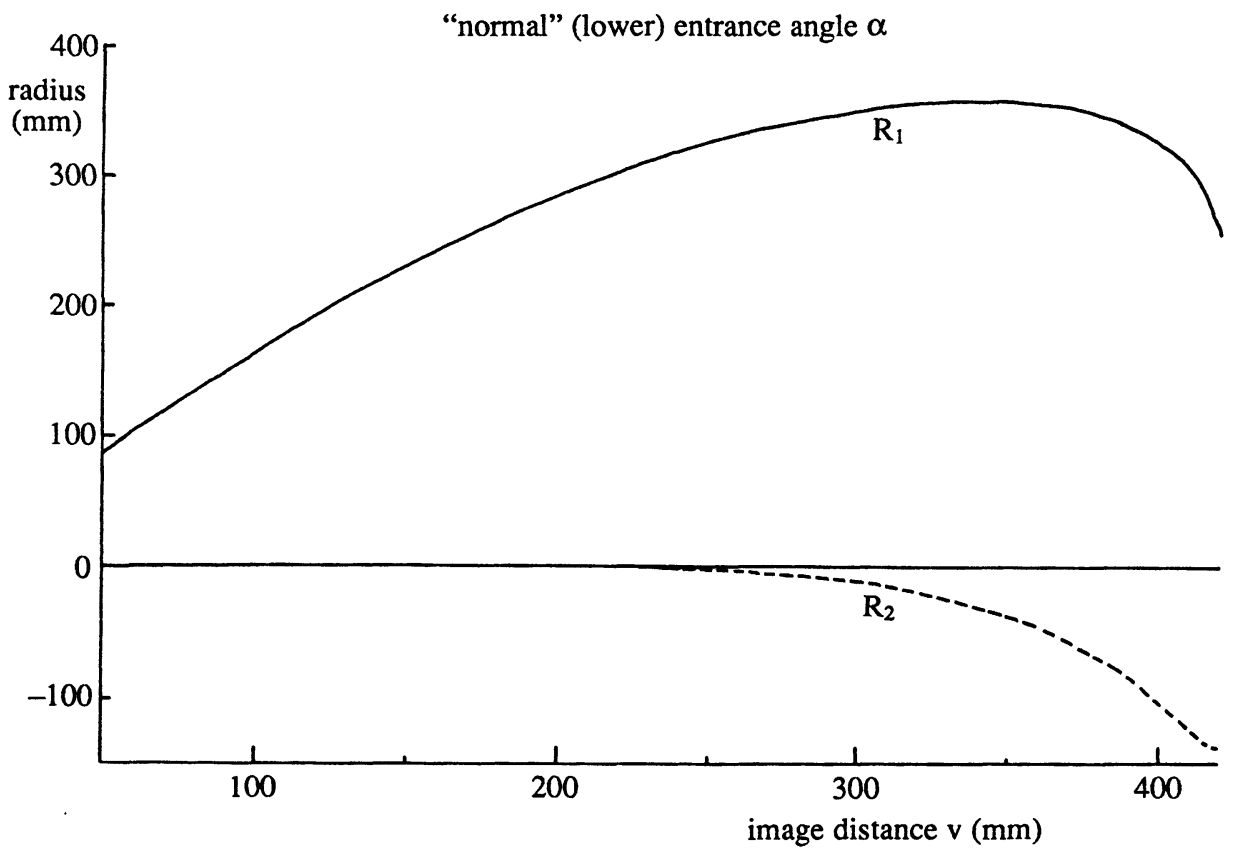

a)

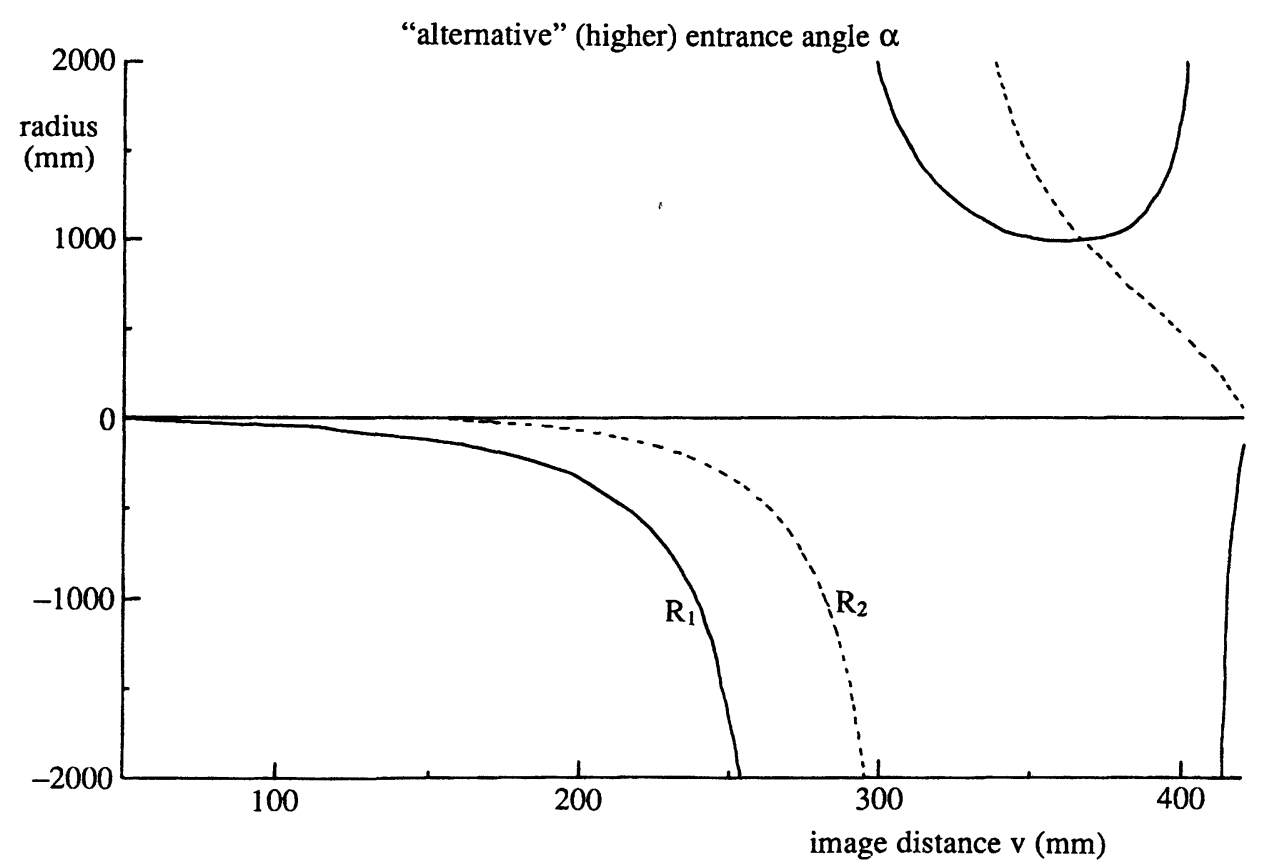

b)

Fig. 3. - Entrance and exit radii required for $Y_{4}$ and $Z_{2}$ to be zero plotted as a function of image distance $(v)$ for a sector alone with a) the "normal" (lower) entrance angle $(\alpha)$ and b) the "alternative" (higher) entrance angle $(\alpha)$. 


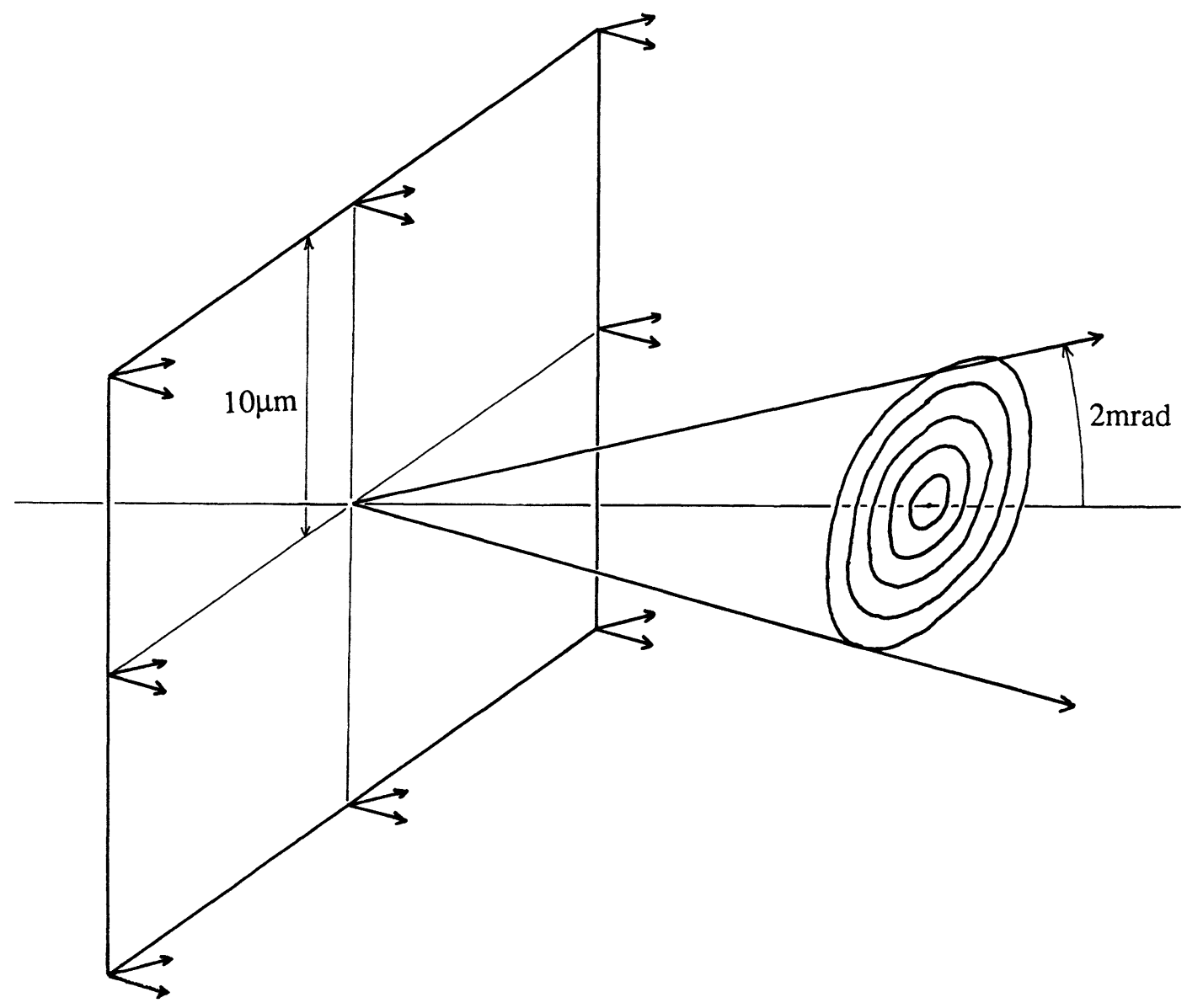

Fig. 4. - Diagram showing the object used for the simulations of the behaviour of a sector shown in figures 5 to 9. It consists of 9 sets of cones of electron trajectories arranged around a square, each set of cones made up of 5 cone trajectories inside each other.

changes of the aberration figure for each of the different energies.

The major imaging effect of the spectrometer is to cause a strong rectangular distortion which is opposite for the two configurations. For the high $\alpha$, low $\beta$ configuration, best for imaging, a square is elongated in the azimuthal direction by 1.7 . Our only major uncertainty in the comparison of the two configurations is that the high $\alpha$ configuration appearing to have better characteristics may be as a result of not considering 3rd order aberrations in the azimuthal direction. In addition, there is a trapeziodal distortion present (see Fig. 9) which cannot, unlike the rectangular distortion, be eliminated using the quadrupoles following the sector (or as in our case following the slit at $\mathrm{A}$ in Fig. 1).

When we remember that a pair of quadrupoles can be used to form a doubly focussed image with a distortion of either of the senses (as shown for the two separate parameter sets) it would appear that the dispersion can be increased prior to the point $\mathrm{A}$ (using the quadrupoles) while decreasing the distortion. At the same time, on the principle of the overall design discussed here it is the quadrupoles after the slit which would be used primarily for the removal of the distortions. Note that the third quadrupole in this part of the chain allows alteration of the dispersion (for 


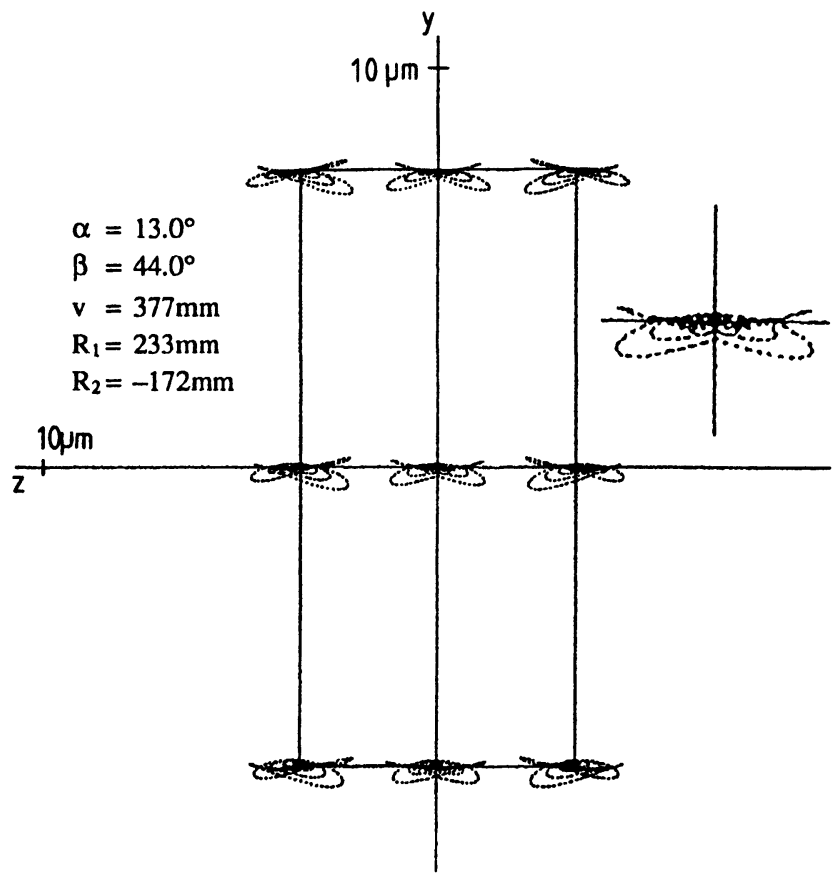

a)

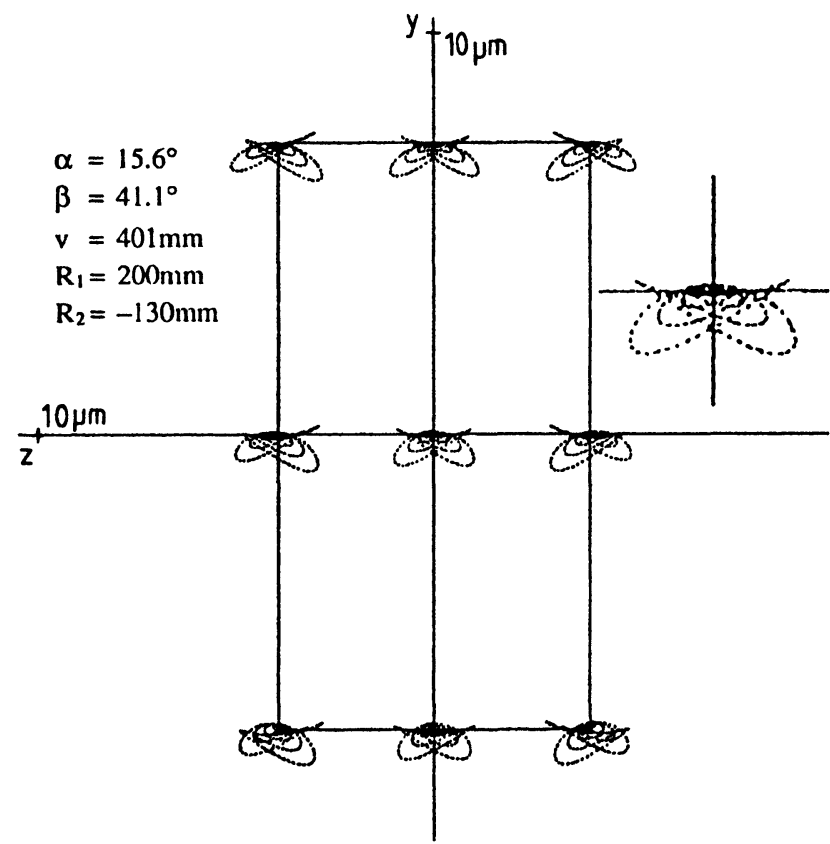

b)

Fig. 5. - The image due to the $\pi / 2$ sector magnet at $V$ when the object shown in figure 4 is imaged with a sector (with no quadrupoles and monochromatic electrons) optimised with low $\alpha$ and high $\beta$ for three image distances, $v$. In an ideal sector with no aberrations the cones of electrons emerging from each of the 9 points at the object would be focussed to a corresponding point in the image of the projector crossover (normally the diffraction pattern) at V. The form of the spread of points shown here indicates the effect of aberrations present in our sector. The image of the central point is shown enlarged at the top right of each figure. The dispersion direction here, as in figures 6 to 9 , is vertical. 


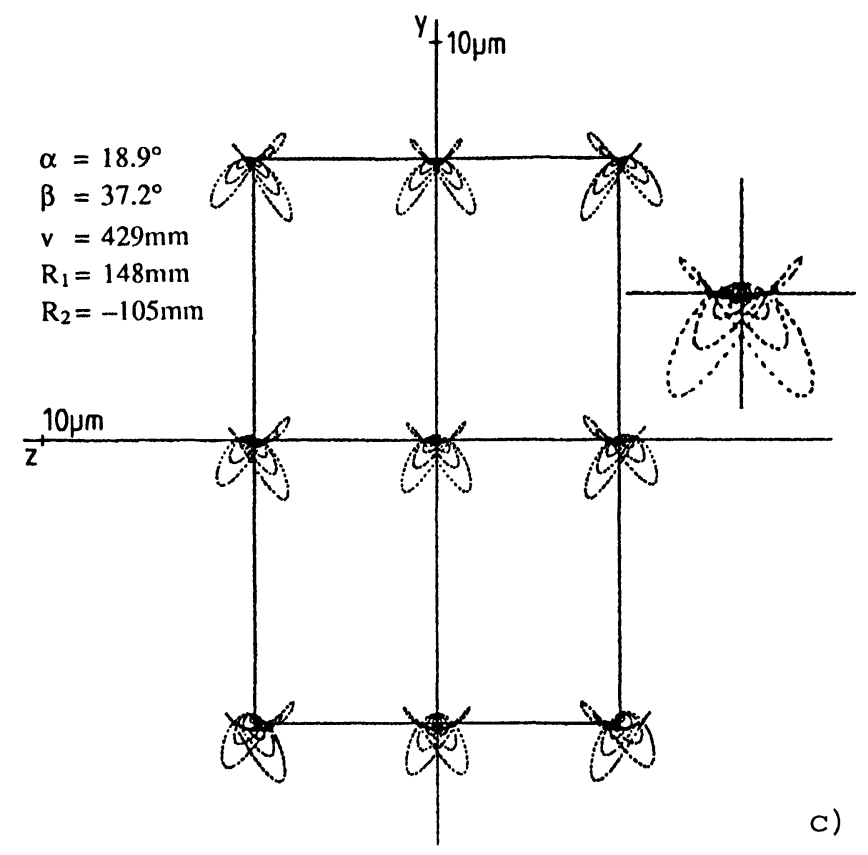

Figure 5 (continued).

energy loss analysis at A). Fundamentally however the trapezoidal distortion caused by the spectrometer (see Fig. 9) can not be eliminated on the approach described. Reconsidering the rectangular distortion after the spectrometer and the first three quadrupoles it is this which should be capable of complete elimination by the second group of quadrupoles. If images are required for different energy dispersions at A (and thus different excitations of the first group of quadrupoles) then the elimination of the rectangular distortion would require changes in the settings of the quadrupoles in the second set.

We have not optimised the design of the system as a whole, but have investigated the various possible combinations of spectrometer and quadrupole groupings which are practical and have concluded that a reasonable imaging system could be achieved, on the basis of the sector magnet design approach described above, with little reduction in the microscope resolution, for slit widths allowing acceptance of electrons with an energy spread of less than about $20 \mathrm{eV}$.

\section{Application of image filters in materials science.}

As we have noted above, given that it is now expected that the Gatan filter [28] will become commercially available within a reasonable period, we will not now proceed to fabricate the system we have described here, but have given a description of some of the work we have done on it, in particular in relation to the magnetic sector, in order to clarify the requirements which can be based on the potential usage of such systems. In order further to emphasise why we feel that filtering is essential, we summarise below the various classes of problem which will become accessible in materials science once an appropriate filter system becomes available.

Firstly it will be clear from section 2 that we do not believe that fully quantitative high resolution imaging is possible without filtering. We can note in this area two types of problem, as also 


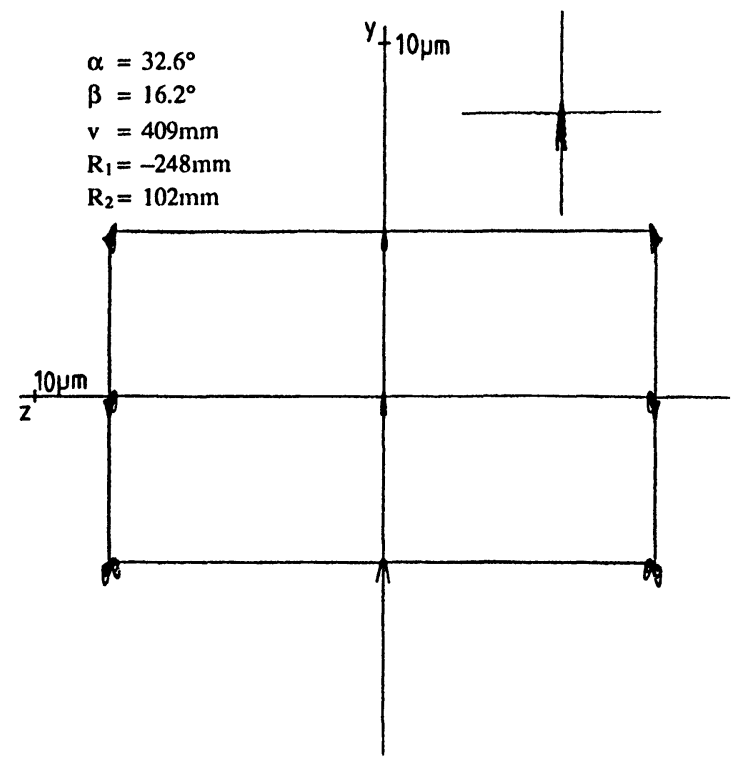

a)

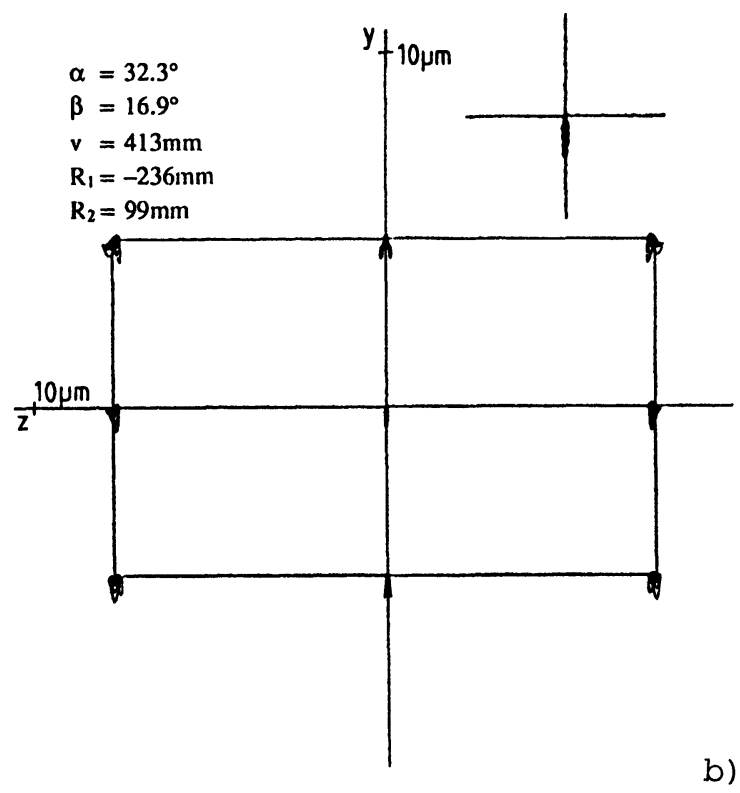

Fig. 6. - As figure 5 but for a sector optimised with high $\alpha$ and low $\beta$. Note that the aberration effects are considerably lower for the alternative than for the normal solution and that now $R_{2}$ is positive.

discussed elsewhere [11], these being essentially the analysis of the relative occupancy of different elements on different sites in the lattice of, for example, a mixed oxide and the detailed structural characterisation of a uniformly projected line or planar defect. We do not of course claim that 

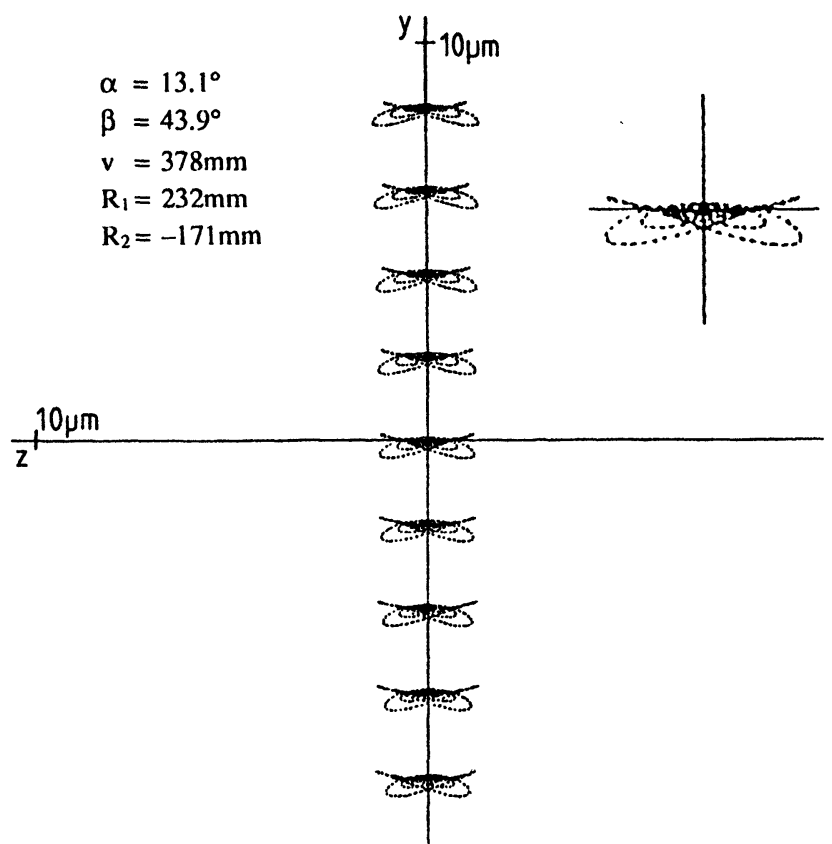

Fig. 7. - The image at $V$ when the central set of cones of electrons now having energies from -4 to 4 Volts in steps of 1 Volt is imaged with a sector optimised with low $\alpha$ and high $\beta$. The electrons with lower energy are deflected upwards (ie in the $+y$ direction). Note the effect of the remnant aberrations in changing the form of the aberration figures as the energy is changed.

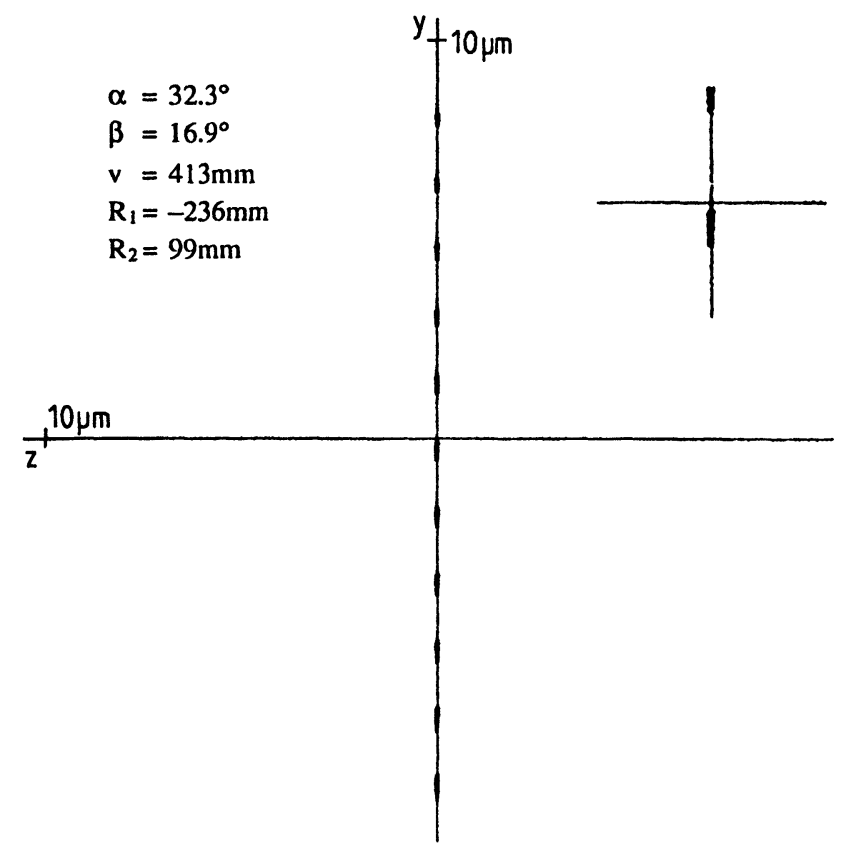

Fig. 8. - As figure 7 but for the high $\alpha$ and $\beta$ case and electron energies from -5 to 5 Volts. 


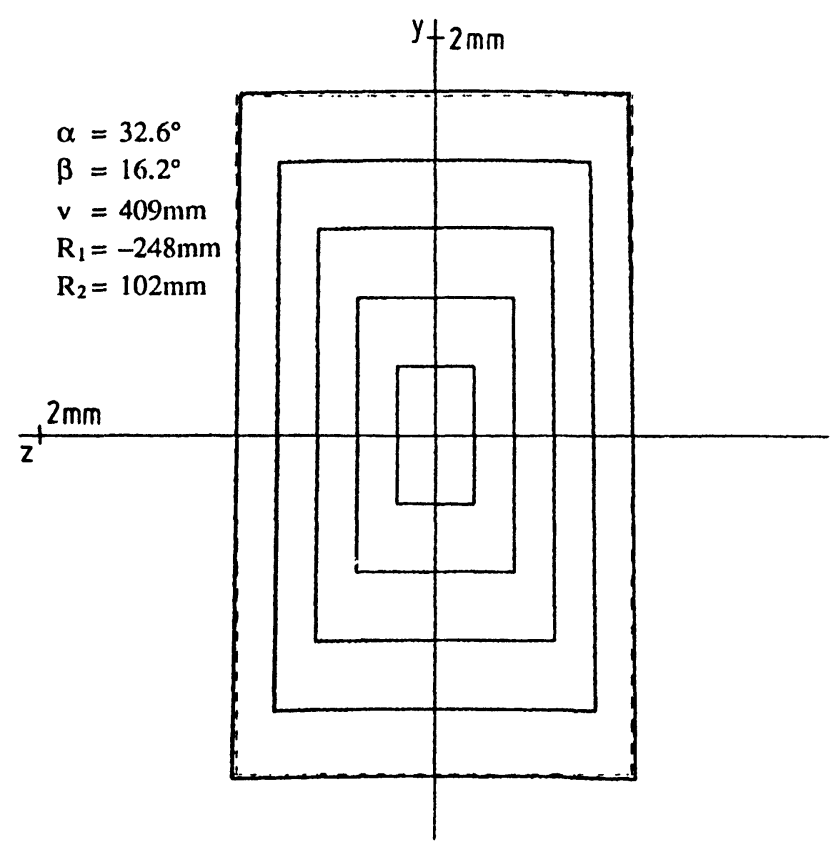

Fig. 9. - The "image" for the sector alone $400 \mathrm{~mm}$ beyond $\mathrm{V}$ when electrons emerging in a set of 5 square based pyramids from the object at the projector crossover are projected with a sector alone (ie no quadrupoles) for the high $\alpha$, low $\beta$ case. This figure gives an idea of both the rectangular distortion, that would be corrected by the duadrupoles, and the remaining distortions, emphasised by the dotted rectangle which would be more difficult to correct.

such problems have no other means of solution (the former problem type above is often amenable to the use of ALCHEMI [31]) but filtering will allow the high resolution approach to be used on a numerically assessable basis rather than essentially only qualitatively as at present.

Secondly it will also be clear that a full analysis of the intensities in the filtered image will allow an elastic scattering factor based compositional analysis to the resolution attained in the filtered image and this should in principle approach that of the microscope. Such filtered images will also improve the accuracy to which the Fresnel Method might be used for local analysis (e.g. [8,9]). The use of specific edges, particularly in difference images, will provide useful checks for these two approaches particularly when there are localised changes in the composition, as for example when there is segregation of a specific element at a grain boundary. It is interesting, incidentally, that the resolution loss which should be noted when boron is finally imaged at a grain boundary in such a manner will provide information on the localisation of the energy loss process rather than on the spatial extent of the segragation. If on the other hand grain boundary segregated phosphorus could be imaged in the weak $\mathrm{K}$ (rather than the more delocalised L) edge then the image, if obtainable at the low intensities available, would provide a measure of the true compositional profile. It is noteworthy that in either case the Fresnel Method would appear to provide the more hopeful approach if it is the segregant's profile that is required.

The third class of probelm, which will require a considerable amount of effort, is the general improvement of a whole range of conventional imaging methods, as currently applied, once filtered images can readily be obtained. Included in this area is the improved analysis of the anomalous contrast in weak beam images of faults (e.g. [32]) and the general improvement in contrast of 
qualitative as well as quantitative applications of the weak beam technique in the area of, for example, stacking fault energy analysis (e.g. [33]).

A fourth area is characterised by the types of problem which can be undertaken once a probe formed convergent beam pattern can be examined both filtered and at different losses. Some of these have been discussed by Cockayne [34] whilst others include the potentially improved analysis of bonding effects (e.g. [35]).

Finally it will be clear that the range of problems which have recently been assessed in the area of surface plasmons (e.g. [36,37]) by specialised high resolution STEM should be as readily, or better approachable, using loss images in TEM.

\section{Acknowledgements.}

We are grateful to Prof. D. Hull for the provision of laboratory facilities and to the Paul Fund of the Royal Society for financial support. We also thank M. Kato and K. Tsuno (JEOL) for their help with some of the calculations.

\section{References}

[1] Ourmazd A., TAYlor D.W., Cunningham J. and Tu C.W., Phys. Rev. Lett. 62 (1989) 933.

[2] HYTCH M.J., BOOTHROYD C.B., ROSS F.M., BITHEL E.G. and STOBBS W.M., Electron Microscopy and Analysis 1989, P.J. Goodhew and H.Y. Elder Eds., IOPCS no 98 (Institute of Physics, Bristol, 1989) p. 345.

[3] STOBBS W.M. and ROSS F.M., Evaluation of advanced semiconductor materials by electron microscopy, D. Cherns Ed., NATO ASI series physics 203 (Plenum, New York, 1989) p. 183.

[4] NeSS J.N., StOBBS W.M. and PAGE T.F., Philos. Mag. A54 (1986) 679.

[5] OZKAYA D., SHIH W.C., NEWCOMB S.B. and STOBBS W.M., Electron Microscopy and Analysis, 1989, P.J. Goodhew and H.Y. Elder Eds., IOPCS no 98 (Institute of Physics, Bristol, 1989) p. 199.

[6] Ross F.M., GIBSON J.M. and STOBBS W.M., MRS syp proc 159 (MRS, Pittsburg, 1989) p. 185.

[7] Ross F.M., BRITTON E.G. and STOBBS W.M., EMAG '87 Analytical Electron Microscopy, G.W. Lorimer Ed. (Institie of Metals, London, 1988) p. 205.

[8] Ross F.M. and STOBBS W.M., Philos. Mag. A63 (1991) 37.

[9] Ross F.M. and STOBBS W.M., Philos. Mag. A63 (1991) 1.

[10] PENNYCOOK S.J. and JeSSON D.E., Phys. Rev. Lett. 64 (1990) 938.

[11] STOBBS W.M. and SAXTON W.O., J. Microsc. 151 (1988) 171.

[12] BOOTHROYD C.B. and STOBBS W.M., Ultramicrosc. 26 (1988) 361.

[13] BOOTHROYD C.B. and STOBBS W.M., Ultramicrosc. 31 (1989) 259.

[14] HYTCH M.J. and STOBBS W.M., EUREM 88, P.J. Goodhew and H.G. Dickinson Eds., IOPCS no 93 (Institute of Physics, Bristol, 1988) p. 347.

[15] Howe A., Proc. Royal Soc. (London) A271 (1963) 268.

[16] HASHIMOTO H., Ultramicrosc. 18 (1985) 19.

[17] STOBBS S.H. and STOBBS W.M., EMAG ' 87 Analytical Electron Microscopy, G.W. Lorimer Ed. (Institite of Metals, London, 1988) p. 111.

[18] REIMER L. and ROSS-MESSEMER M., J. Microsc. 155 (1989) 169.

[19] REIMER L. and ROSS-MESSEMER M., J. Microsc. 159 (1990) 143.

[20] BAKENFELDER A., Fromm I., REIMER L. and RENNEKAMP R., J. Microsc. 159 (1990) 161.

[21] CundY S.L., METHERELl A.J.F. and WHELAN M.J., J. Scient. Instrum. 43 (1966) 712.

[22] Cundy S.L., METHERELl A.J.F. and WhELAN M.J., Philos. Mag. 15 (1967) 623.

[23] Rose H. and Plies E., Optik 40 (1974) 336.

[24] SHUMMAN H., Ultramicroscopy 5 (1980) 45.

[25] KRIVANeK O.L., Proc EMSA, G.W. Bailey Ed. (Claitors, Baton Rouge, 1979) p. 530.

[26] SHuman H., Chang C.-F. and SOMLYO A.P., Ultralicrosc. 19 (1986) 121. 
[27] Bourdillon A.J., Stobbs W.M., Page K., Home R., Wilson C., Ambrose B., Turner L.J. and TEBBY G.P., Electron Microscopy and Analysis 1985, G.J. Tatlock Ed., IPOCS no. 78 (Adam Hilger, Bristol, 1985) p. 161.

[28] GUBBENS A.J. and KRIVANEK O.L., presented at Lake Tahoe Workshop on EELS, 17-22 August 1990, Take Tahoe, California, USA and O.L. Krivanek and C. Ahn, Proc XIth Int Cong on EM, Kyoto 1986, T. Imura, S. Maruse and T. Suzuki Eds.(Japanese Society of Electron Microscopy, Tokyo, 1986) p. 519.

[29] ENGE H.A., Chapter 4.2 in "Focussing of Charged Particles" vol. II, A. Septier Ed. (Academic Press, New York, 1967).

[30] EGERTON R.F., Electron Energy Loss Spectroscopy in the Electron Microscope (Plenum Press, New York, 1986).

[31] TAFTØ J. and SPENCE J.C.H., Ultramicrosc. 9 (1982) 243.

[32] Bithell E.G., DONOVAN P.E. and STOBBS W.M., Phil. Mag. 59A (1989) 63.

[33] STOBBS W.M., Electron microscopy in materials science, E. Rüedl and U. Valdré Eds. (comm of Eur Comm, 1975) p. 594.

[34] COCKAYNE D.J.H. and McKENZIE D.R., EUREM 88, P.J. Goodhew and H.G. Dickinson Eds., IOPCS no 93 (Institute of Physics, Bristol, 1988) p. 17.

[35] SPENCE J.C.H., ZUO J.M. and HOIER R., Evaluation of Advanced semiconductor materials by electron microscopy D. Cherns Ed., NATO ASI Series Phys. 203 (Plenum, New York, 1989) p. 101.

[36] WANG Z.L. and COWLEY J.M., Ultramicroscopy 23 (1987) 97.

[37] HOWIE A. and MILNE R.H., Ultramicroscopy 18 (1985) 427. 